\title{
Editorial
}

\section{Optimization Theory, Methods, and Applications in Engineering 2014}

\author{
Jung-Fa Tsai, ${ }^{1}$ John Gunnar Carlsson,, ${ }^{2}$ Dongdong Ge, ${ }^{3}$ Yi-Chung Hu, ${ }^{4}$ and Jianming Shi ${ }^{5}$ \\ ${ }^{1}$ Department of Business Management, National Taipei University of Technology, No. 1, Section 3, Chung-Hsiao East Road, \\ Taipei 10608, Taiwan \\ ${ }^{2}$ Program in Industrial and Systems Engineering, University of Minnesota, 111 Church Street SE, Minneapolis, MN 55455, USA \\ ${ }^{3}$ Research Center for Management Science and Data Analytics, School of Information Management and Engineering, \\ Shanghai University of Finance and Economics, Shanghai 200433, China \\ ${ }^{4}$ Department of Business Administration, Chung Yuan Christian University, Chung-Li 320, Taiwan \\ ${ }^{5}$ School of Management, Tokyo University of Science, 500 Shimokiyoku, Kuki, Saitama 346-8512, Japan
}

Correspondence should be addressed to Jung-Fa Tsai; jftsai@ntut.edu.tw

Received 12 July 2015; Accepted 12 July 2015

Copyright (C) 2015 Jung-Fa Tsai et al. This is an open access article distributed under the Creative Commons Attribution License, which permits unrestricted use, distribution, and reproduction in any medium, provided the original work is properly cited.

Optimization theory and methods have been applied in various fields to handle various practical problems over the past few decades. Many approaches have been proposed to solve engineering optimization problems, and the methods can be divided into deterministic and heuristic ones. With the advances in computing techniques and the increasing reliance on optimization-based approaches in real applications, optimization techniques have continuously received attention from the practitioners and the researchers and many novel approaches have been proposed in the last few years. This special issue aims to present some recent developments in the area of optimization theory, methods, and applications in engineering. This special issue involves 17 original papers, selected by the editors and related to the various researches themes on optimization theory, methods, and applications in engineering. According to the characteristics of the accepted papers, the special issue is organized as the following three parts and each part is composed of several important papers to the part's scope.

Deterministic Optimization. W. Si et al. present a novel direction of arrival estimation method in compressed sensing. The proposed method is derived through transforming quadratically constrained linear programming into unconstrained convex optimization. Their method overcomes the drawback that $l_{1}$-norm is nondifferentiable when sparse sources are reconstructed by minimizing $l_{1}$-norm. The convergence rate and estimation performance of the proposed method can be significantly improved, since the steepest descent step and Barzilai-Borwein step are alternately used as the search step in the unconstrained convex optimization. Simulation results show the superior performance of the proposed method as compared with existing methods.

"Energy-Saving Generation Dispatch Using Minimum Cost Flow" by Z. Zhang and X. Cai uses a minimum cost flow method to solve a dispatch problem in order to minimize the consumption of coal in the dispatching of a thermal power system. The minimum cost flow problem, considering the loss of network flow, is known as a generalized network flow problem, which can be expressed as a quadratic programming problem in mathematics. Theoretical analysis and numerical results prove the correctness and effectiveness of the proposed method.

W. Wei, et al. propose a new technique of vehicle handling inverse dynamics which can evaluate the emergency collision avoidance performance. Based on the optimal control theory, the steering angle input and the traction/brake force imposed by a driver are the control variables; the minimum time required to complete the fitting biker line change is the control object. By using the improved direct multiple shooting method, the optimal control problem is converted 
into a nonlinear programming problem that is then solved by means of the sequential quadratic programming. The simulation results show that the proposed method can solve the vehicle minimum time maneuver problem and can compare the maneuverability of two different vehicles that complete fitting biker line change with the minimum time, and the correctness of the model is verified through real vehicle test.

"Robust Control of the Knee Joint Angle of Paraplegic Patients considering Norm-Bounded Uncertainties" by $\mathrm{N}$. M. dos Santos et al. presents a proposal for the knee position control design of paraplegic patients with functional electrical stimulation using control systems and considering norm-bounded uncertainties. A state-space representation of the knee joint model of the paraplegic patient with its nonlinearity is demonstrated and the use of linear matrix inequalities in control systems with norm-bounded uncertainties for asymptotic stability is analyzed.

S. Choi and K. Park study a few dynamic risk-averse inventory models using additive utility functions. They add Markovian behavior of purchasing costs in the models and provide the problem formulations with finite and infinite MDP (Markovian Decision Process) problems. For finite time models, they first prove (joint) concavity of the model for each state and obtain a (modified) base-stock optimal policy. Then, they conduct comparative static analysis for model parameters and derive monotone properties to the optimal solutions. For infinite time models, they show the existence of stationary base-stock optimal policies and the inheritance of the monotone properties proven at the finite time models.

X. Xian-hao et al. investigate the capacity allocation policies of a third-party warehouse center, which supplies several different level services on different prices with fixed capacity, on revenue management perspective. For the singleperiod situation, they use three different robust methods, absolute robust, deviation robust, and relative robust, to maximize the whole revenue. For the multiperiod situation, as the demand is uncertain, they propose a stochastic model for the multiperiod revenue management problem of the warehouse. A novel robust optimization technique is applied to maximize the whole revenue in the proposed model. Then some numerical examples are given to verify the practical applicability of their method.

The paper "Equilibrium Customer Strategies in the Queue with Threshold Policy and Setup Times" by P. Chen et al. considers the equilibrium behavior of customers in the $\mathrm{M} / \mathrm{M} / 1$ queue with $\mathrm{N}$ policy and setup times and addresses the equilibrium threshold strategies for the fully observable case and mixed strategies for the fully unobservable case, respectively. This study also gets various performance measures of the system and investigates some numerical examples of system size, social benefit, and expected cost function per unit time for the two different cases under equilibrium customer strategies.

Heuristic Algorithms. The paper "Metaheuristic Approaches for Solving Truck and Trailer Routing Problems with Stochastic Demands: A Case Study in Dairy Industry" by S. Mirmohammadsadeghi and S. Ahmed aims at modeling the truck and trailer routing problem with stochastic demand constraints to bring the truck and trailer routing problem model closer to a reality. The model is solved in a reasonable timeframe using data from a large dairy service by administering the multipoint simulated annealing, memetic algorithm, and tabu search. A sizeable number of customers whose demands follow the Poisson probability distribution are considered to model and solve the problem. Experimental results indicate that the average results obtained by the proposed memetic algorithm (MA) are improved about 1.27 and 0.78 percent comparing with tabu search and multipoint simulated annealing, respectively. However, the differences between the results are insignificant. Consequently, the algorithms are found to be appropriate and relevant for solving the real-world truck and trailer routing problem with stochastic demands.

I. Hwang et al. propose a new heuristic that identifies clusters in the population of partitions which have already been optimized locally. The clusters must be created to seed the memetic algorithms, and as the memetic algorithm runs it makes the beneficial cluster moves. The research results on standard benchmark graphs show significant reductions in cut size and get better solutions than those in the literature for some cases.

"Maintaining Track Continuity for Extended Targets Using Gaussian-Mixture Probability Hypothesis Density Filter" by Y. Han et al. presents a multiextended target tracker based on the extended target Gaussian-mixture probability hypothesis density filter to provide the tracks of the extended targets in maintaining the track continuity for the extended targets. To improve the performance of the extended target tracker, the study also proposes a mixture partitioning algorithm for resolving the identities of the extended targets in close proximity. The simulation results show that the proposed tracker achieves the less error of the position estimates and decreases the probability of incorrect label assignments.

"A Variable Depth Search Algorithm for Binary Constraint Satisfaction Problems" by N. Bouhmala presents a fast metaheuristic for solving binary constraint satisfaction problems. The method can be classified as a variable-depth search metaheuristic combining a greedy local search using a self-adaptive weighting strategy on the constraint weights. Experiments are conducted on hard random constraint satisfaction problems enjoying several features that make them of a great theoretical and practical interest. The results show that the proposed metaheuristic is capable of solving hard unsolved problems that still remain a challenge for both complete and incomplete methods. In addition, the proposed metaheuristic is remarkably faster than all existing solvers when tested on previously solved instances. Finally, its distinctive feature contrary to other metaheuristics is the absence of parameter tuning making it highly suitable in practical scenarios.

The paper "An Effective Hybrid of Bees Algorithm and Differential Evolution Algorithm in Data Clustering" by M. B. Bonab et al. is to minimize the dissimilarity of all points of a cluster, from gravity center of the cluster with respect to capacity constraints in each cluster, such that 
each element is allocated to only one cluster. This article presents a new hybrid algorithm based on cluster center initialization algorithm (CCIA), bees algorithm (BA), and differential evolution (DE), known as CCIA-BADE-K, aiming at finding the best cluster center. The evaluation results of the proposed algorithm and its comparison with other alternative algorithms in the literature confirm its superior performance and higher efficiency.

P. Liang et al. consider an unrelated parallel machine scheduling problem with energy consumption and total tardiness. They establish a mathematical model for this problem and then present an ant optimization algorithm based on ATC heuristic rule (ATC-ACO). Furthermore, the optimal parameters of the proposed algorithm are defined via Taguchi methods for generating test data. Finally, the results of comparative experiments indicate the proposed ATC-ACO algorithm has better performance on minimizing energy consumption as well as total tardiness and the modified ATC heuristic rule is more effective on reducing energy consumption.

Information System and Multicriteria Decision-Making. "A Real-Time Pothole Detection Approach for Intelligent Transportation System" by H.-W. Wang et al. proposes a pothole detection method based on the mobile sensing. The accelerometer data is normalized by Euler angle computation and is adopted into the pothole detection algorithm to obtain the pothole information. Moreover, the spatial interpolation method is used to reduce the location errors from global positioning system data. The experimental results show that the proposed approach can precisely detect potholes without false-positives, and the higher accuracy is performed by the proposed approach. Therefore, the proposed real-time pothole detection approach can be used to improve the safety of traffic for intelligent transportation system.

The paper "Parallel Control to Fragments of a Cylindrical Structure Driven by Explosive inside" by W. Chen et al. presents a new design method for the shell shape. To facilitate the establishment of the numerical model and the machining for relative experiments, the mathematical description of the theoretical calculated generatrix of the shell is simplified. Based on the theoretical design and plentiful simulation data, the relationships between the size of the parallel fragmentation structure and the optimized curvature radius of the shell are expressed by an equation. The equation is validated by numerical means and can be a reliable reference to the design of the parallel fragmentation structure.

"To Make Good Decision: A Group DSS for Multiple Criteria Alternative Rank and Selection" by C.-S. Wang et al. proposes a group decision support system architecture named hybrid decision-making support model (HDMSM), integrating four decision approaches (Delphi, DEMATEL, ANP, and MDS) to help the decision maker in ranking and selecting appropriate alternatives. The HDMSM consists of five steps, namely, criteria identification, criteria correlation calculation, criteria evaluation, critical criteria selection, and alternative rank and comparison. According to the case study demonstration, the proposed HDMSM can enable a group of decision makers to implement the MCDM effectively and help them to analyze the relation and degree of mutual influence among different evaluation factors.

The paper "Configuration, Deployment, and Scheduling Models for Management and Optimization of Patrol Services" by B. Yang et al. presents a decision support system (DSS) and its models for patrol service center (PSC). This work contributes to the literature on patrol services and network optimization problems in the following aspects: based on a series of models, a DSS framework is designed for PSCs; the models are formulated for resource management and scheduling upon geography information system; coordination strategies among close PSCs are incorporated into decision models. These features are examined in integration manners. The assessment criteria and optimization models studied in the paper are beneficial for building DSSs for PSC.

Jung-Fa Tsai

John Gunnar Carlsson Dongdong $G e$ $\mathrm{Yi}$-Chung $\mathrm{Hu}$ Jianming Shi 


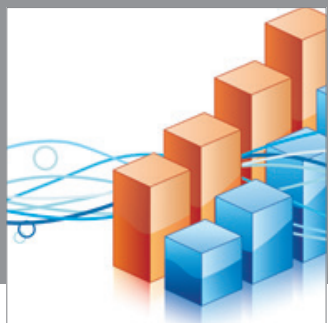

Advances in

Operations Research

mansans

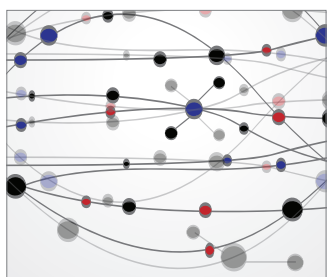

The Scientific World Journal
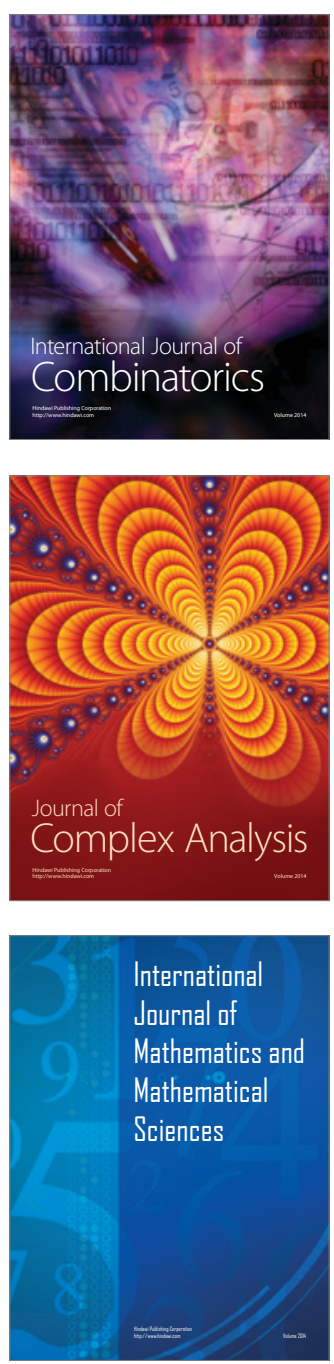
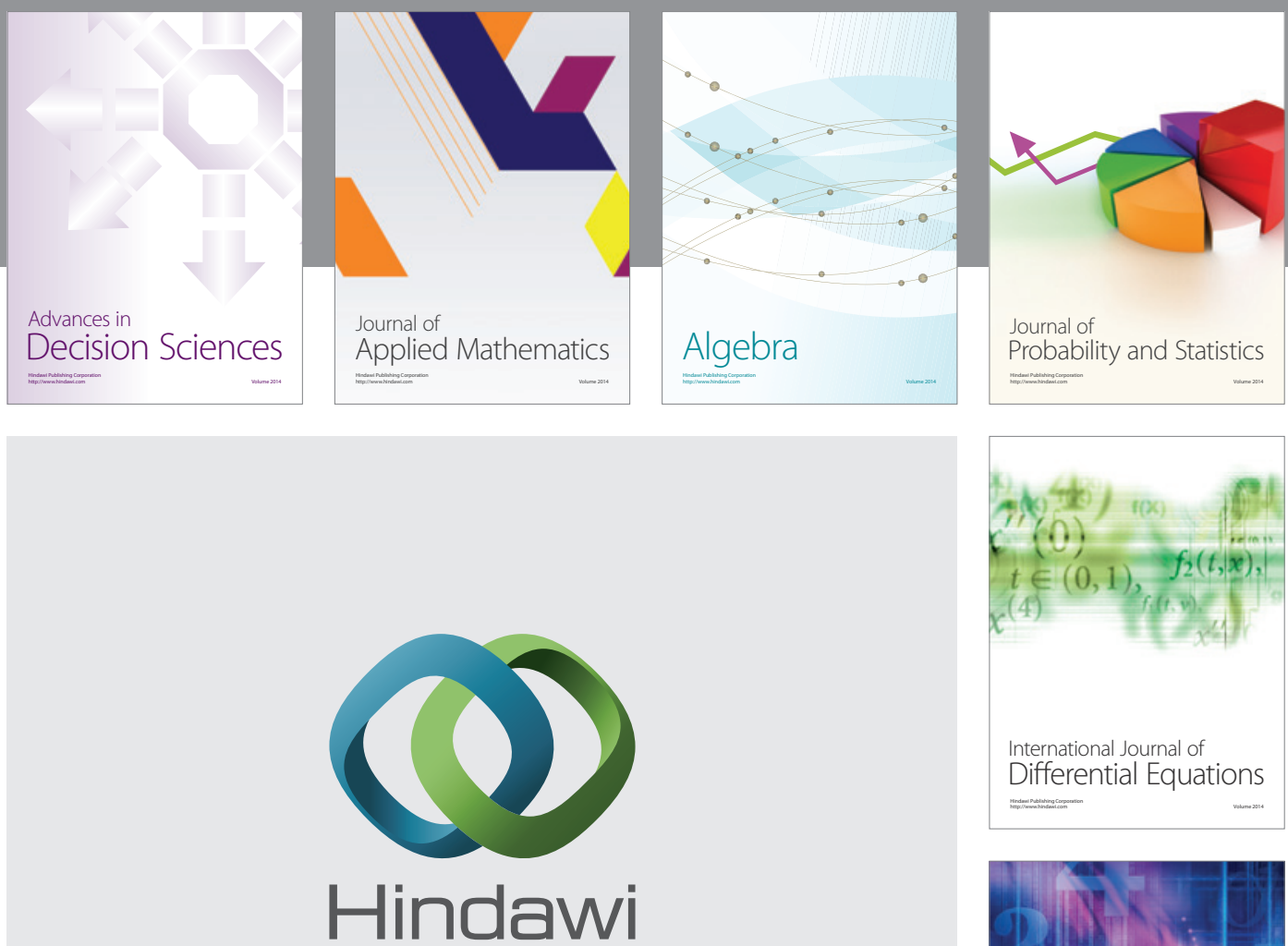

Submit your manuscripts at http://www.hindawi.com
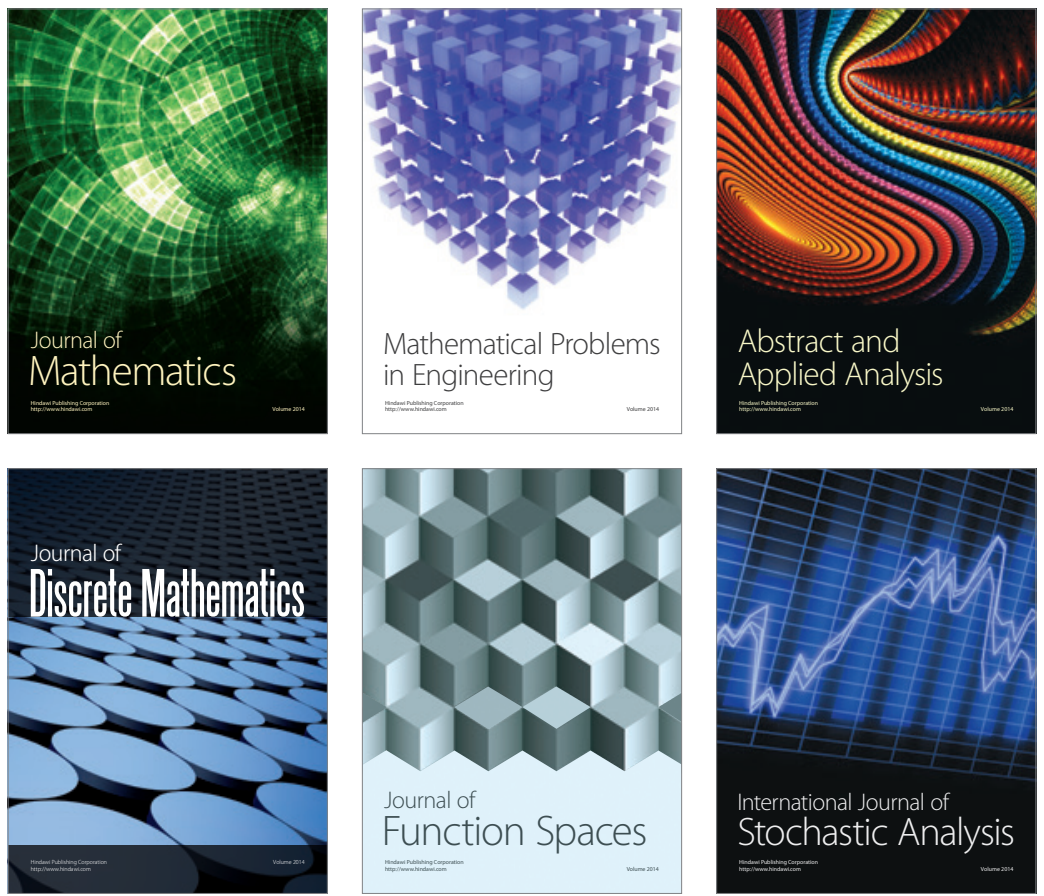

Journal of

Function Spaces

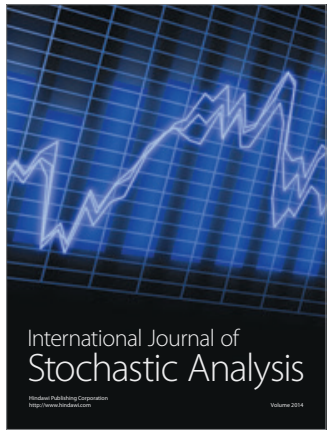

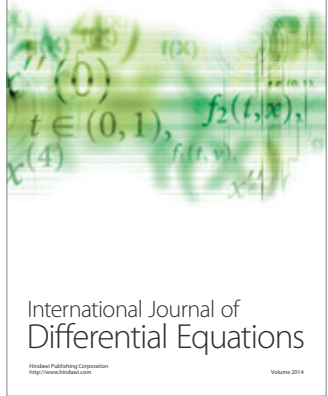
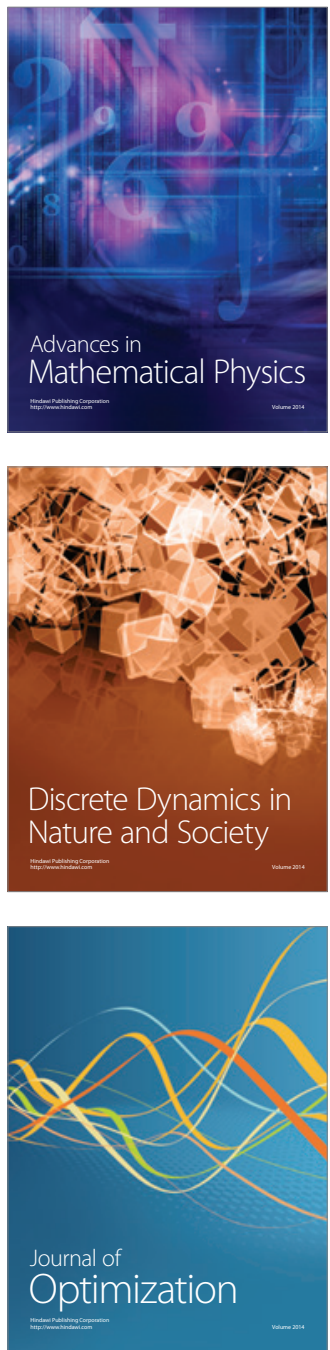